\title{
Effect of methylimidazole on cellulose/ionic liquid solutions and regenerated material therefrom
}

\author{
Carina Olsson • Artur Hedlund • Alexander Idström • \\ Gunnar Westman
}

Received: 22 November 2013/Accepted: 15 January 2014/Published online: 28 January 2014

(C) The Author(s) 2014. This article is published with open access at Springerlink.com

\begin{abstract}
Cellulose, especially wood-based cellulose, is increasingly important for making everyday materials such as man-made-regenerated textile fibers, produced via dissolution and subsequent precipitation. In this paper, the effect of cosolvents in ionic liquid-facilitated cellulose dissolution is discussed. Both microcrystalline cellulose and dissolving grade hardwood pulp were studied. Three different cosolvents in combination with ionic liquid were evaluated using turbidity measurements and viscosity. The ionic liquid precursor $\mathrm{N}$-methylimidazole proved to be a promising cosolvent candidate and was thus selected for further studies together with the ionic liquid 1-ethyl-3methylimidazolium acetate. Results show that dissolution rate can be increased by cosolvent addition, and the viscosity can be significantly reduced. The solutions were stable over time at room temperature and could be converted to regenerated textile fibers with good mechanical properties via airgap spinning and traditional wet spinning.
\end{abstract}

\section{Olsson · G. Westman $(\bowtie)$}

Organic Chemistry, Department of Chemical and Biological Engineering, Chalmers University of Technology,

41296 Göteborg, Sweden

e-mail: westman@chalmers.se

C. Olsson

e-mail: carina.olsson@chalmers.se

\section{A. Hedlund}

Swerea IVF AB, 43122 Mölndal, Sweden

e-mail: artur.hedlund@swerea.se

\author{
A. Idström \\ Applied Surface Chemistry, Department of Chemical and \\ Biological Engineering, Chalmers University of Technology, \\ 41296 Göteborg, Sweden \\ e-mail: idstrom@chalmers.se
}

Fibers spun from binary solvents exhibited significantly higher crystallinity than the fibers from neat ionic liquid.

\section{Introduction}

Due to its structure, cellulose is insoluble or only partly soluble in most common solvents. From the amphiphilic appearance of the glucose unit structure, both hydrogen bonds and hydrophobic interactions arise and the solvent of choice should be able to break both of these in order to dissolve the polymer completely [1,2].

Ionic liquids (ILs) are organic salts with melting points below $100{ }^{\circ} \mathrm{C}$, and sometimes even below room temperature. The possibility to pair anions with cations yields an almost endless library of ILs, and they are used in numerous fields, such as separation technology, electrochemistry, catalysis, and as solvents [3, 4]. In the last decade, ILs as solvents for biomass in general and cellulose in particular have been widely investigated [5-7] since 2002 when Swatloski published the pioneering work on dissolution of cellulose in 1-butyl-3-methylimidazolium chloride (BMIMCl) [8]. This relatively new solvent class has shown great versatility in the field of cellulose technology, including dissolution for regeneration purposes [9, 10], homogeneous derivatization $[11,12]$, and biomass processing including wood component separation [13-15]. The ILs that are able to dissolve cellulose include several classes of cations and a multitude of anions. Some of the most common cations are imidazolium, pyridinium, ammonium, and phosphonium derivatives [16]. Although imidazolium-based ILs are known to form covalent bonds with cellulose in some cases $[17,18]$, they are still among the most used in academic research on ILs and cellulose and still highly interesting for biomass processing [19]. 
Cosolvents can be added to ILs to modify properties of the solvent and/or resulting solution. It has been suggested that appropriate cosolvents can be found by measuring solvatochromic parameters [20], which has been reported for e.g., $\mathrm{N}$-methylimidazole (MIM) [21]. Dimethylsulfoxide (DMSO), $\mathrm{N}, \mathrm{N}$-dimethylacetamide (DMAc), and dimethylformamide (DMF) have been used together with BMIMCl and other ILs to increase reactivity and/or processability of cellulose in solution [22, 23]. Cosolvents can be used to facilitate derivatization, e.g., molecular bases such as pyridine used as catalysts in tosylation reactions [21] and for viscosity modification [24]. Several other molecular solvents have been used as cosolvents together with 1-ethyl-3-methylimidazolium acetate (EMIMAc) for cellulose dissolution, and it has been concluded that amide-type cosolvents allow for cellulose dissolution even when only a small amount of IL is present, typically less than $30 \mathrm{~mol} \%$ of the solvent mixture [25]. The cosolvent is thus used as the bulk in the solvent system, and the IL is used as an additive that adjusts the solvent properties just enough to allow dissolution over merely swelling. MIM as an additive in small amounts has been studied in solutions of BMIMCl and cellulose and was found to drastically reduce DP loss in cotton pulp, and the authors describe a hypothesis of cellulose/BMIMCl/MIM interaction where MIM withdraws some of the chloride ions from the cellulose, thereby inhibiting chain scission [26]. MIM directly facilitates certain reactions and may be successfully used as a cosolvent or additive in derivatization [21, 27]. In fact, it has been shown that MIM, which is a precursor for many imidazolium-based ILs, is quite necessary for some reactions to take place at all [28].

The present study focuses on MIM as a cosolvent to modify the viscosity of cellulose solutions in the ILs EMIMAc and BMIMCl. To the best of our knowledge, no broad study including viscometry and dissolution rates for cellulose in this interesting cosolvent has been done. We study the solubility of cellulose in ILs in the presence of MIM and, for comparison, other cosolvents (DMAc and DMSO). The optimal ratio of different components in the solution, as well as dissolution rate as a function of cosolvent concentration and stability toward degradation over time in the presence of cosolvent, is discussed. Furthermore, regenerated cellulose in the form of filament fibers is prepared from binary solvents including MIM and evaluated.

\section{Experimental}

\section{Materials}

Microcrystalline cellulose (Avicel PH101, Fluka, $M_{\mathrm{w}}=$ $\left.53470, M_{\mathrm{n}}=24235\right)$, used as a model substance in initial experiments, and dissolving grade hardwood pulp from Eucalypt (HWP, Bahia A/S, $M_{\mathrm{w}}=217142, M_{\mathrm{n}}=73394$ ), used for regenerated fiber spinning, were dried at $60{ }^{\circ} \mathrm{C}$ overnight and then stored in a desiccator until use. MIM (Sigma Aldrich, $\geq 99 \%$ ), DMSO (Sigma Aldrich, $\geq 99.5 \%$ ), DMAc (Sigma Aldrich, $\geq 99.5 \%$ ), EMIMAc (BASF, $\geq 90 \%$, LOT STBC9122V), and BMIMCl (Sigma Aldrich, $99 \%$ ) were stored dry and used without further purification. Only very small water content $(<0.3 \%)$ was detected by Karl Fisher titration in the ILs.

\section{Method}

Sample preparation

Cellulose was dispersed in cosolvent (MIM, DMSO, or DMAc) in desired ratio and was allowed to stand for 2-3 min in a sealed vessel. Subsequently, the IL was added and the vessel content was mixed. For the sample without cosolvent, cellulose was dispersed directly in IL. Samples were then heated at $50{ }^{\circ} \mathrm{C}$ for $5 \mathrm{~h}$.

The molar fraction $x_{\mathrm{IL}}$ of IL in the solvent is expressed as

$x_{\mathrm{IL}}=\frac{n_{\mathrm{IL}}}{n_{\mathrm{IL}}+n_{\text {cosolvent }}}$

where $n_{\mathrm{IL}}$ and $n_{\text {cosolvent }}$ denote the amount of substance for the IL and cosolvent, respectively.

Samples were considered fully dissolved if the solution appeared clear by focused beam reflectance measurement (FBRM) measurements and/or by turbidity measurements.

Cellulose solubility and dissolution rate

Apparent absorbance of MCC solutions was measured at 800, 825, and $849 \mathrm{~nm}$ on an Agilent 8453 (G1103A) UV/ vis spectrometer using the UV-Visible ChemStation software and recalculated to turbidity according to

$\tau=\operatorname{Abs} \cdot \frac{\ln 10}{L}$

where Abs is the apparent absorbance as an average of the chosen wavelengths and $L$ is the cuvette length. These wavelengths were chosen, because in this interval there is no actual molecular absorbance of the solvent which means that all signal detected should originate from light scattering, i.e., turbidity arising from undissolved particles in the sample. Calibration curves for solubility determination were constructed by measuring turbidity as apparent absorbance of known amounts of microcrystalline cellulose dispersed in pure cosolvent. For comparison, micrographs for selected solutions were captured on a Zeiss Discovery 
V12 microscope using a Canon Powershot G9 camera mounted on a Zeiss universal digital camera adapter $\mathrm{d} 30$ $\mathrm{M} 37 / 52 \times 0.75$.

Dissolution rate was studied in situ using an FBRM probe (FBRM G600, Mettler Toledo) to track the number of MCC particles in solution as a function of time. Data were collected every $3 \mathrm{~s}$ and analyzed using the iC FBRM software. Cellulose was dispersed in MIM to record a baseline prior to IL addition when applicable.

\section{Flow properties assessment}

Complex viscosity $\left(\eta^{*}\right)$ and zero shear viscosity $\left(\eta_{0}\right)$ measurements were performed in triplicates on an oilheated Bohlin instruments CS50 rheometer equipped with $4 \% / 40 \mathrm{~mm}$ stainless steel cone and plate geometry at $30{ }^{\circ} \mathrm{C}$ and/or $70{ }^{\circ} \mathrm{C}$ for MCC samples and $8 \mathrm{wt} \%$ pulp samples and with $5.44 \% 25 \mathrm{~mm}$ stainless steel cone and plate geometry at $30{ }^{\circ} \mathrm{C}$ and $70{ }^{\circ} \mathrm{C}$ for the $15 \mathrm{wt} \%$ pulp samples. The measurements were performed in a closed cell to avoid moisture uptake in the hygroscopic samples.

\section{Intrinsic viscosity}

Specific viscosity $\left(\eta_{\mathrm{sp}}\right)$ of cellulose was calculated from three replicates of flow time measurements in a set of capillary viscometers at $25^{\circ} \mathrm{C}$ for a series of increasingly dilute cellulose solutions in different EMIMAc/MIM mixtures. Intrinsic viscosity $([\eta])$ was then calculated as the limiting value of $\eta_{\mathrm{sp}}$ from $\eta_{\mathrm{sp}} / \mathrm{C}$ versus $\mathrm{C}$ extrapolated to zero cellulose concentration, where $\mathrm{C}$ is the cellulose concentration in $\mathrm{g} \mathrm{ml}^{-1}$. It was assumed that $\eta_{\mathrm{sp}}+1=\eta /$ $\eta_{0} \approx t / t_{0}$, where $\eta / \eta_{0}$ represents viscosity of the solution divided by viscosity of the solvent, and $t / t_{0}$ represents flow time of the solution divided by flow time of the solvent. Measurements were performed on very dilute microcrystalline cellulose samples, less than $1 \mathrm{wt} \%$, stated to be well below the overlap concentration for cellulose in this DP range [29, 30]. Not all samples of interest could be studied by this method, as the viscosity of the solvent mixture containing mostly IL was too high. In this case, capillary viscometry is not applicable and those samples had to be omitted.

\section{Degree of polymerization}

Degree of polymerization was estimated for microcrystalline cellulose Avicel PH-101 and the eucalypt pulp used in fiberspinning trials by size exclusion chromatography via dissolution in DMAc and $8 \% \mathrm{LiCl}$ and subsequent dilution with DMAc to a final $\mathrm{LiCl}$ concentration of $0.5 \%$ and a cellulose concentration of $0.4 \mathrm{mg} \mathrm{ml}^{-1}$. The samples were separated by molecular weight on a set of three columns, PLgel Mixed A
$(300 \times 7.5 \mathrm{~mm})$ with a guard column (Polymer Laboratories Ltd.), and analyzed using a refractive index detector HP 1047 (Hewlett-Packard). Temperature of the columns was set to

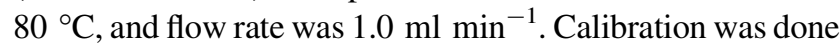
using narrow polystyrene standards with molecular weights ranging from 5120 to $6120000 \mathrm{Da}$.

Fiber spinning and evaluation

Airgap- and wet spinning of Eucalypt dissolving grade pulp in EMIMAc/MIM solvents were performed at Swerea IVF, Mölndal, Sweden. All solutions were heated at $70{ }^{\circ} \mathrm{C}$ and filtered through a $20 \mu \mathrm{m}$ sintered stainless steel wire nonwoven filter placed at the exit of the spin-dope container, from which the heated solution was pressed by the descending piston during spinning. Although heated prior to passing through the filter, the solution was extruded at room temperature as it flowed slowly through a distance of ambient temperate piping which caused it to cool down prior to extrusion. Two different spinnerets were used, with 100 holes of the diameter $80 \mu \mathrm{m}$ or 31 holes with the diameter of $150 \mu \mathrm{m}$ (in the case of pure EMIMAc, which could not be extruded within the pressure limits of the equipment, since ambient temperature was used for the extrusion). For the solutions containing cosolvent, spun through the $80 \mu \mathrm{m}$ spinneret extrusion, speed was set at $3 \mathrm{~m} \mathrm{~min}^{-1}$ for the $15 \mathrm{wt} \%$ cellulose solutions and $10 \mathrm{~m} \mathrm{~min}^{-1}$ for the $8 \mathrm{wt} \%$ cellulose solutions, while the pure EMIMAc/cellulose solution was spun at $2.8 \mathrm{~m} \mathrm{~min}^{-1}$ (150 $\mu \mathrm{m}$ capillaries).

When applied, the air gap was set to between 6 and $10 \mathrm{~mm}$. Coagulation took place in water, and fibers were drawn by rotating take-off rollers to desired extent. The fibers were then immersed in pure water for 1 week before drying at $105{ }^{\circ} \mathrm{C}$ for $1 \mathrm{~h}$. All samples were equilibrated at the testing conditions $T=20 \pm 2{ }^{\circ} \mathrm{C}$ and $\mathrm{RH}=65 \pm 2 \%$ before titer (linear density) and stress-strain curves of dry fibers were determined using a Vibroskop/Vibrodyn instrument (Lenzing Technik $\mathrm{GmbH} \& \mathrm{Co} \mathrm{KG).} \mathrm{Clamp} \mathrm{distance} \mathrm{was} \mathrm{set} \mathrm{to} 20 \mathrm{~mm}$, and testing speed was $20 \mathrm{~mm} \mathrm{~min}^{-1}$. Data from tensile testing are presented as averages from ten experiments, with standard deviations.

Quantitative CP/MAS NMR experiments were performed as previously described elsewhere [31].

\section{Results and discussion}

Solubility and solution viscosity for cellulose in binary solvents

Absorbance measurements to determine solubility are only valid in a narrow region where a linear relationship can be 
established. Nevertheless, in this case, it is sufficiently sensitive to probe transition from the insoluble to the soluble region of cellulose in IL/cosolvent mixture. The calibration curves show linearity below a turbidity value of around $3.5 \mathrm{~cm}^{-1}$, where it can be translated into an amount of undissolved material in the solution according to the calibration curves in the "Appendix" section. By knowing the amount of undissolved material and the amount of cellulose added to the sample, the amount of solubilized cellulose is known and this value can in turn be recalculated into solubility in percent for each solution with a known composition.

Figure 1 shows the solubility of MCC in EMIMAc/ cosolvent solutions with different compositions. All solutions contained ten percent cellulose by weight. It is obvious that at an IL concentration corresponding to an IL to AGU ratio of between one and two, there is an abrupt transition from a state where a significant amount of undissolved material is present, to a clear solution when using MIM or DMSO as the cosolvent. DMAc, which is the bulk component in the widely used $\mathrm{DMAc} / \mathrm{LiCl}$ solvent system, proves not the most feasible in combination with EMIMAc. As seen in Fig. 1, it takes at least twice as much IL to dissolve cellulose when using DMAc as a cosolvent in comparison to when using MIM, a molar ratio IL to AGU of around 3.3 compared to 1.4 .

Interestingly, when using MIM together with BMIMCl as the active solvent component, $10 \mathrm{wt} \%$ MCC was not soluble in BMIMCl/MIM until xIL reached 0.5, corresponding to a molar ratio nIL/nAGU of 5.7. This result was valid regardless of temperature, in the range between 50 and $110^{\circ} \mathrm{C}$. For this reason, BMIMCl was left out from further studies.

For flow properties, the samples were characterized by means of rotational viscometry after dissolution for $5 \mathrm{~h}$. Some of the results are plotted in Fig. 1 and show a prominent decrease in viscosity as an effect of decreased ratio of IL, for all three cosolvents. The viscosity of the molecular solvents is considerably less than for the IL, and thus, the viscosity of the solvent mixture is a function of the ratio of EMIMAc/MIM. It can be noted in Fig. 1 that the different cosolvents have different effects on the viscosity at concentrations around the solubility threshold of cellulose. For MIM, the viscosity is steadily decreasing as a function of decreased IL concentration $\left(n_{\mathrm{IL}} / \mathrm{nAGU}\right)$, even beyond the point where cellulose does not dissolve completely according to turbidity measurements. When using DMSO as a cosolvent, the viscosity increases dramatically even before the solubility limit, i.e., cellulose is dissolved completely on the macroscopic level as indicated by the turbidity measurements, but on a molecular level there seems to be aggregation and/or gelation causing increased viscosity. The results for samples with DMAc as a
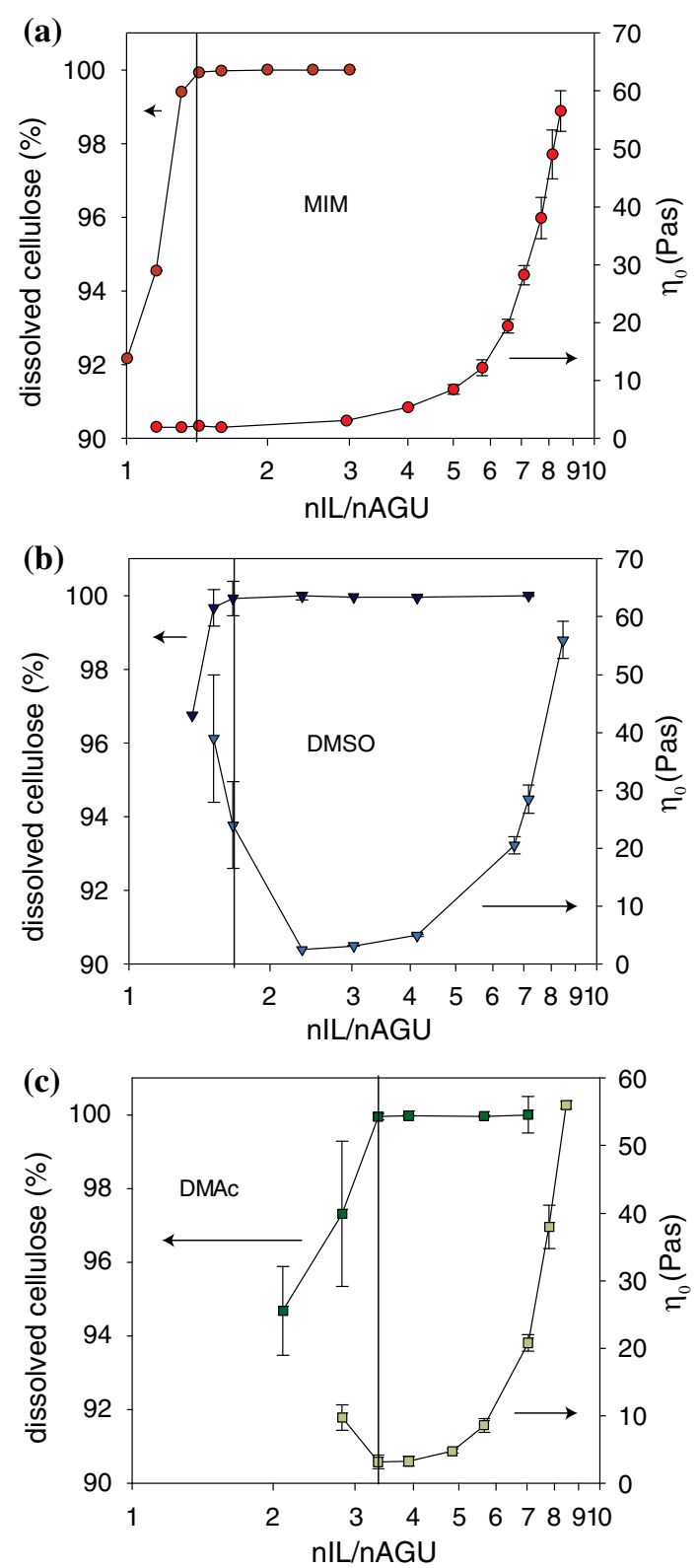

Fig. 1 Solubility and zero shear viscosity at $30{ }^{\circ} \mathrm{C}\left(\eta_{0}\right)$ of $10 \mathrm{wt} \%$ $\mathrm{MCC}$ as a function of the molar ratio of ionic liquid to cellulose (AGU) when using a MIM, b DMSO, or c DMAc as cosolvent. Lines are provided to guide the eye. The vertical line in each plot represents apparent solubility limit according to the turbidity measurements

cosolvent show something in between; the viscosity increases at lower concentrations of IL, but only for nondissolved samples. Turbidity data are consistent with micrographs of cellulose solutions as in Fig. 2 in the IL to AGU ratio of interest, i.e., around the point where dissolution takes place. The transition from a turbid to a clear solution is obvious when comparing picture (a) and (c), where picture (a) shows $10 \%$ MCC in EMIMAc and MIM where the amount of EMIMAc is not sufficient to dissolve the polymer $\left(n_{\mathrm{IL}} / \mathrm{nAGU}=1.16\right)$ and picture $(\mathrm{c})$ shows fully dissolved polymer $\left(n_{\mathrm{IL}} / \mathrm{nAGU}=1.43\right)$. 

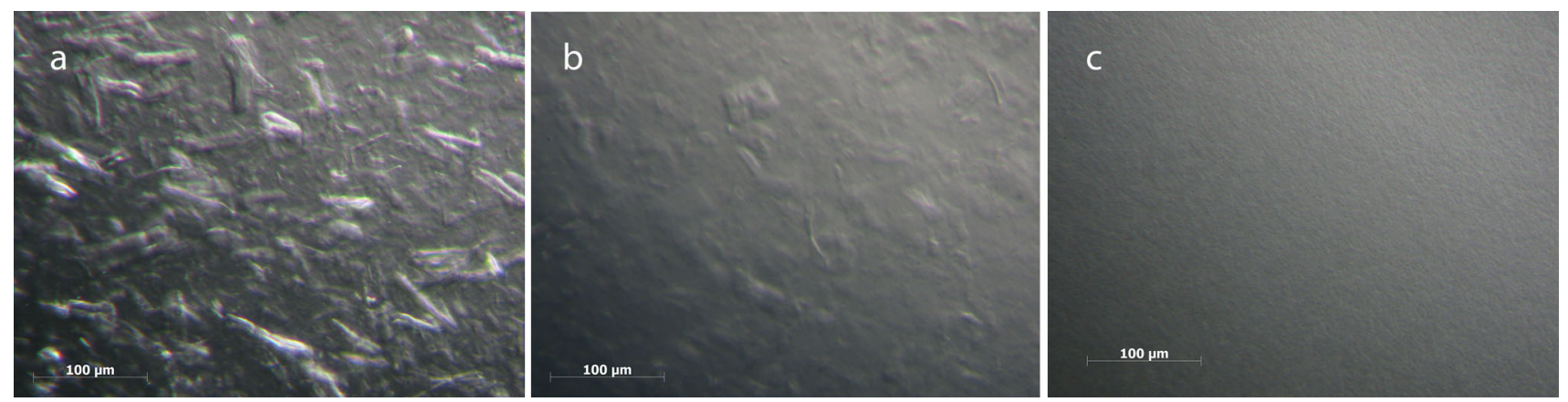

Fig. 2 Micrographs showing the transition from non-dissolved to dissolved state of $10 \% \mathrm{MCC}$ when increasing the IL ratio from a $n_{\mathrm{IL}} /$ $\mathrm{nAGU}=1.16 ; x_{\mathrm{IL}}=0.07$ to $\mathbf{b} n_{\mathrm{IL}} / \mathrm{nAGU}=1.31 ; x_{\mathrm{IL}}=0.08$ and $\mathbf{c} n_{\mathrm{IL}} / \mathrm{nAGU}=1.43 ; x_{\mathrm{IL}}=0.09$

As shown, the amount of EMIMAc required for dissolving microcrystalline cellulose corresponded to $\sim 1.4$ mol EMIMAc per mol AGU using MIM as the cosolvent. Other cosolvents tested did not reach the same low threshold; for DMSO and DMAc, the observed corresponding thresholds were 1.5 and 3.3, respectively. These results can be compared with literature data where the molar ratio $\left(x_{\mathrm{IL}}\right)$ in the solvent mixture was 0.08 using DMSO and 0.27 using DMAc, which corresponds to molar ratios $n_{\mathrm{IL}} / \mathrm{nAGU}$ of 1.4 and 6.8, respectively [25]. Solutions of $10 \mathrm{wt} \%$ cellulose with different EMIMAc concentrations $\left(x_{\mathrm{IL}}\right)$ and attempts to maximize the cellulose content in solutions of fixed xIL ratios both resulted in the conclusion that the molar ratio $n_{\mathrm{IL}} / \mathrm{nAGU}$ must exceed at least $\sim 1.4$ for dissolution of MCC to take place, even at very low cellulose concentration, but increasing concentration of MCC demands increasing relative amounts of EMIMAc, as seen in Fig. 3a. In contrast to MCC, where solubility is largely depending on cellulose concentration, Eucalypt dissolving pulp was soluble in the EMIMAc/MIM mixture with nIL/nAGU exceeding 2.4 in a wide concentration range, as seen in Fig. 3b. This value corresponds well to that previously reported for DMSO [32]. For both cosolvents, this indicates by extrapolation that the maximum amount of cellulose that is soluble in pure EMIMAc is close to $28 \mathrm{wt} \%$, similar to what has been estimated using NMR methods [33].

\section{Cellulose conformation: intrinsic viscosity}

The decreased viscosity of the solutions with small amount of IL is not only due to the decreased solvent viscosity, but most likely also a result of change in polymer to solvent interaction, i.e., "solvent quality". With reduced amount of acetate anions in the solvent mixture, there is a reduced possibility of solvent-polymer hydrogen bonding and an increased possibility of polymer to cosolvent or polymer to polymer interaction, the latter ultimately leading to precipitation. The intrinsic viscosity of cellulose in solutions of EMIMAc/MIM was studied to understand the polymer conformation in the different solvent mixtures. Results of these measurements are presented in Fig. 4 and show an increase in hydrodynamic radius of cellulose as the amount of EMIMAc is increased.

Intrinsic viscosity here describes the volume occupied by the cellulose in solution which is directly dependent on the interactions between polymers and solvent [34]. In this case, that corresponds to the solvation potential of the solvent mixtures of EMIMAc/MIM. The same method was used recently to monitor solvent-cellulose interaction for IL with water impurities [35]. As seen in Fig. 4, intrinsic viscosity increases with increasing amount of active solvent component, i.e., EMIMAc, meaning that the dissolution capacity of the solvent is directly linked to the IL concentration as can be expected when mixing a solvent and a non-solvent. This continuous change in solvent quality contrasts significantly to the results from turbidity measurements for macroscopic solubility determination where a distinct threshold can be noted. Due to the low cellulose concentration in the intrinsic viscosity measurements, all samples are far above the critical ratio $n_{\mathrm{IL}} / \mathrm{nAGU}$ threshold where dissolution takes place; even the sample with the lowest concentration of IL has around $18 \mathrm{~mol}$ of IL per mol of AGU for the $1 \%$ cellulose solutions, more than ten times as much of the active solvent component that is required for dissolution according to turbidity measurements. In other words, even though a small amount of IL is enough to get the polymer in solution at the macroscopic scale, a higher amount of active solvent component (EMIMAc) will influence solvent quality even beyond the point of actual dissolution. At the same time, it was observed that once a cellulose sample was dissolved in EMIMAc/MIM-mixed solvent, it could be diluted with large volumes of MIM without any visible sign of precipitation and it seems in this case that the molar ratio $x_{\mathrm{IL}}$ is less important than the ratio $n_{\mathrm{IL}} / \mathrm{nAGU}$.

\section{Dissolution rate}

Dissolution rate as measured by an FBRM probe for the dissolution of microcrystalline cellulose in EMIMAc/MIM 


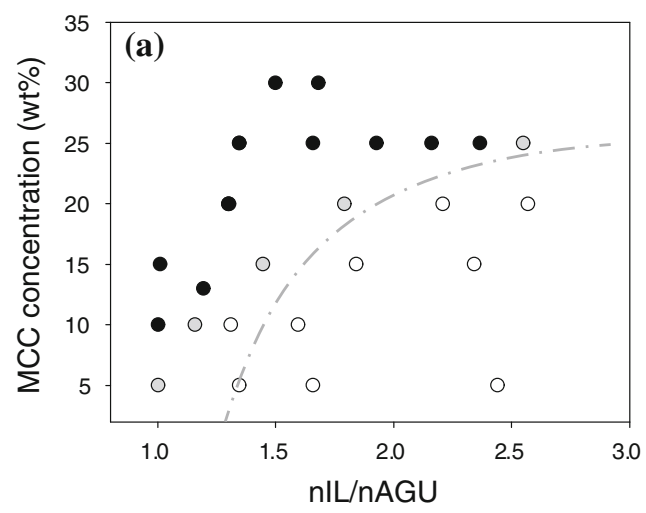

Fig. 3 Solubility of a MCC and b Bahia pulp in EMIMAc/MIM binary solvent at $50{ }^{\circ} \mathrm{C}$ as determined by microscopy at different concentrations of ionic liquid in the solvent, expressed as moles EMIMAc per mol anhydroglucose unit ( $\left.n_{\mathrm{IL}} / \mathrm{nAGU}\right)$. The symbols are

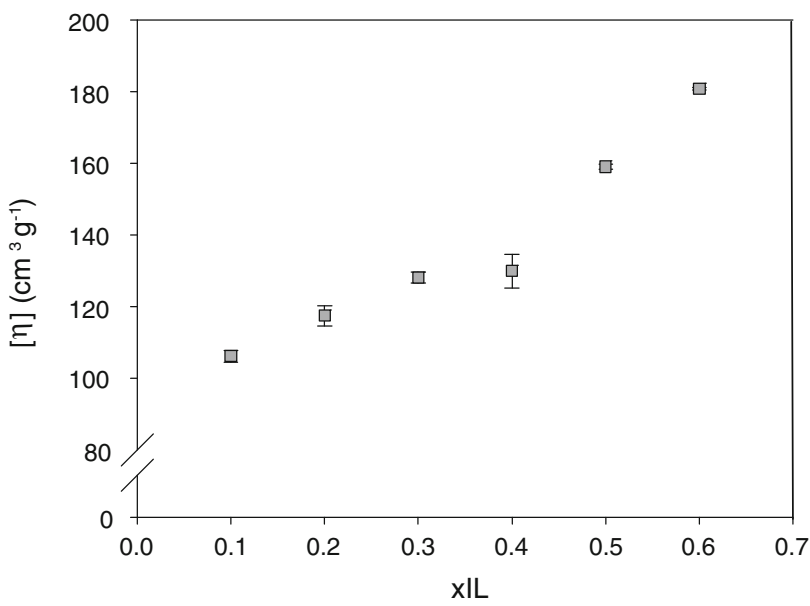

Fig. 4 Intrinsic viscosity of cellulose in EMIMAc/MIM solutions as a function of ionic liquid content in the solvent. The values are based on tree replicates for each data point

are given in Fig. 5. It can be seen that by using MIM as a dispersion agent and cosolvent, the rate of dissolution increased significantly, as indicated by the arrow; clear solutions of $10 \%$ cellulose formed within a few minutes under moderate stirring for the sample with the lowest amount of IL $\left(x_{\mathrm{IL}}=0.1\right)$, in contrast to the much slower dissolution of cellulose in pure IL $\left(x_{\mathrm{IL}}=1\right)$. The increase in dissolution rate at lower amounts of IL seen in the inset in Fig. 5 in this case is most likely a direct consequence of reduced viscosity and enlarged surface area exposed to the solvent due to dispersion prior to dissolution. The FBRM probe measures chord length distribution on micrometer scale which can be complicated to interpret quantitatively in the case of needle-like particles such as cellulose crystals assuming some measurements will be along the particle and some will be across. Identical data processing for all

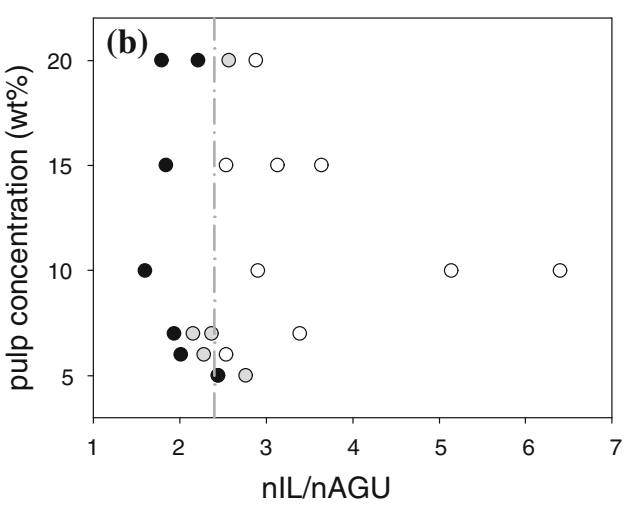

read as follows. Black dots: non-dissolved samples, gray dots: single or few fragments remaining, white dots: no visible fragments remaining. The dotted lines corresponds to the apparent solubility limit of cellulose

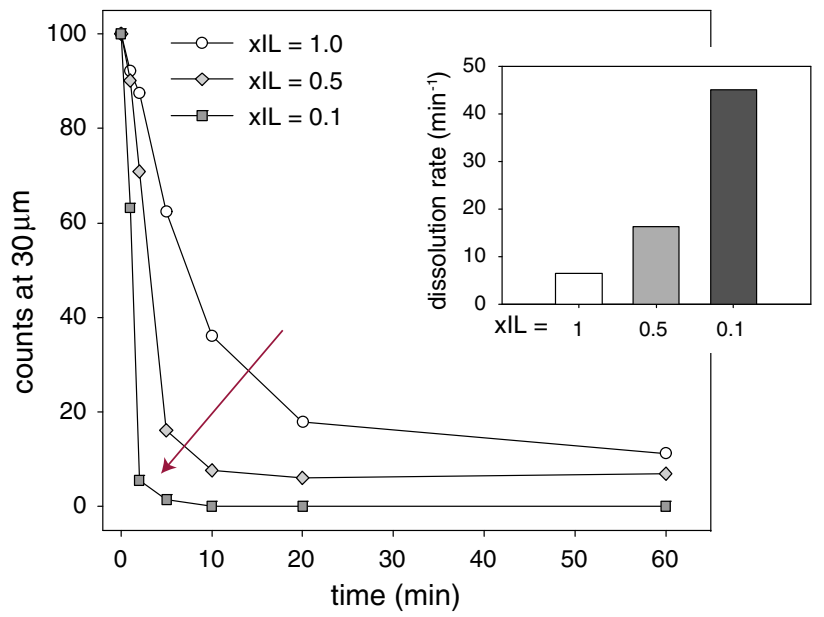

Fig. 5 FBRM data of particle counts at $30 \mu \mathrm{m}$ for $10 \% \mathrm{MCC}$ solutions in EMIMAc/MIM expressed as percent of the initial value at $t=0$ in solutions with IL molar ratio of $1.0,0.5$, and 0.1 . Lines are provided to guide the eye. Inset shows initial dissolution rate extracted from the FBRM data

samples must be ensured, and the results cannot be quantitatively compared to e.g., spherical particles.

The interactions between methylimidazole and cellulose are too weak to cause dissolution, but it is likely that the low amount of EMIMAc needed to dissolve cellulose in this mixed solvent system is due to that MIM is actually facilitating dissolution and prevents chain aggregation. In comparison to the other cosolvents used in this study, MIM allowed for the least amount of IL being used. We interpret this as MIM being a suitable cosolvent in this sense, although the results for the more intensively studied DMSO are very close, as seen in Fig. 1. Computer simulations has shown that imidazole, which is analogous to MIM in its molecular structure, interacts with the glucose ring by 
hydrophobic stacking in an aqueous solution, primarily on the H1-H3-H5-side of the glucose ring, and in addition might also act as a weak hydrogen bond acceptor [36]. Methylimidazole should exhibit the same type of interactions with cellulose as imidazole without the disadvantage of being a solid. Similar results has been shown in studies of imidazolium cation interactions with glucose [36], but in the case of dissolution the much stronger anion-glucose hydrogen bond interaction will dominate and finally cause dissolution [37]. As a consequence of the weak nature of the MIM-cellulose interaction and the low viscosity of MIM, the increased dissolution rate for the mixture of EMIMAc/MIM compared to pure EMIMAc should mainly be understood as a result of increased mobility; dispersion of cellulose in a non-solvent with low viscosity prior to dissolution is known to facilitate mass transport by increasing solid liquid interface as the non-solvent is replaced by solvent. This procedure is used in the NMMO process, where cellulose is dispersed in water which is later evaporated to a certain concentration that allows dissolution, and a similar technique has also been used for water/ IL binary mixtures [10].

\section{Cellulose stability in binary solvent mixtures}

The impact of EMIMAc/MIM ratios on the degradation of cellulose in solutions of EMIMAc/MIM was briefly investigated. The degradation studies were done on $10 \mathrm{wt} \%$ solutions at room temperature. The degradation seems moderate, as clearly seen in Table 1; sample 2 and sample 3 showed only a small DP loss after 31 days of storage, $1.4 \%$ DP loss with $x_{\mathrm{IL}}=0.9$, and a $3 \%$ DP loss with $x_{\mathrm{IL}}=0.1$. These results suggest that cellulose solutions in EMIMAc/MIM are relatively stable over time toward degradation at room temperature.

Fiber spinning from binary-mixed solvents

Five different samples were prepared for regeneration by airgap- and wetspinning techniques. For all samples containing cosolvent (MIM), an $80-\mu \mathrm{m}$ holes spinneret was used. For the samples dissolved in neat EMIMAc, the viscosity was too high and the spinneret had to be equipped with holes of $150 \mu \mathrm{m}$ in diameter. Viscosity data of all samples are found in the "Appendix" section. Fiber tensile testing data, Fig. 6, clearly show that it is possible to maintain the same mechanical properties, while reducing the fiber linear density, when using the binary solvent system. As the exact velocities measured from godets turning in the lower part of their range of possible speeds are often poor in accuracy, the amount of stretch (in the air
Table 1 Degradation of cellulose in MIM/EMIMAc solutions after 31 days storage in room temperature, measured by GPC. Sample 1 is the starting material

\begin{tabular}{llll}
\hline Sample no. & Sample description & $\mathrm{DP}_{n}$ & DP loss \\
\hline 1 & MCC & 150 & - \\
2 & MCC,$x_{\mathrm{IL}}=0.1$ & 145 & $3 \%$ \\
3 & MCC,$x_{\mathrm{IL}}=0.9$ & 148 & $1.4 \%$ \\
\hline
\end{tabular}

gap) is best deduced from the filament titer measured (the dope feed rate was kept constant). In Fig. 6, the actual titers are on the $\mathrm{x}$-axis corresponding to the inverse of stretch for all data except for the three data points from the $150 \mu \mathrm{m}$. In Fig. 7b, these data points have been normalized (factor of 64/225) by the capillary cross-section area, making the titer of the whole set of data points comparable with regard to stretch, i.e., the titer on $x$-axis is inversely proportional to the amount of stretch applied (zero stretch corresponding to $10 \mathrm{dtex}$ ). Most striking then is how relatively compact the data points are along possible trend lines (even though from different $x_{\mathrm{IL}}$ compositions), particularly for the elastic modulus and elongation to break. Figure $7 \mathrm{a}$ features the typical compromise between strain to break (ductility) on one hand and tenacity and stiffness on the other. It is clear that very good elongation can be achieved with the use of cosolvent if stretch is kept moderate. However, these fibers are only comparable to

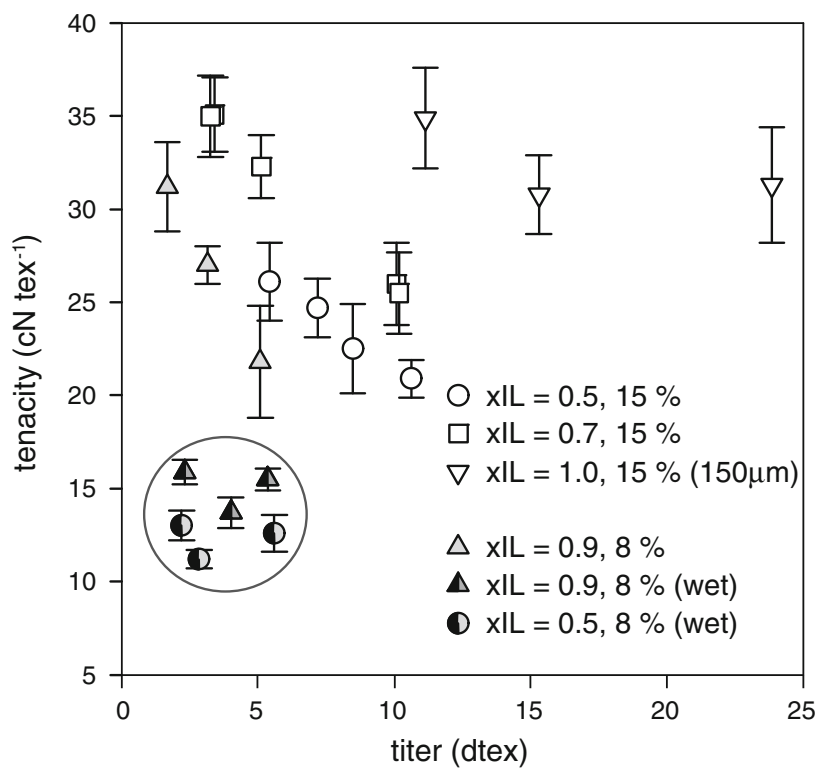

Fig. 6 Tensile testing fiber data for all fibers spun from EMIMAc/ MIM solutions. The encircled samples are from wet spinning. Note that the sample without cosolvent (white triangles) was extruded through a spinneret with larger holes, $150 \mu \mathrm{m}$ instead of $80 \mu \mathrm{m}$ 


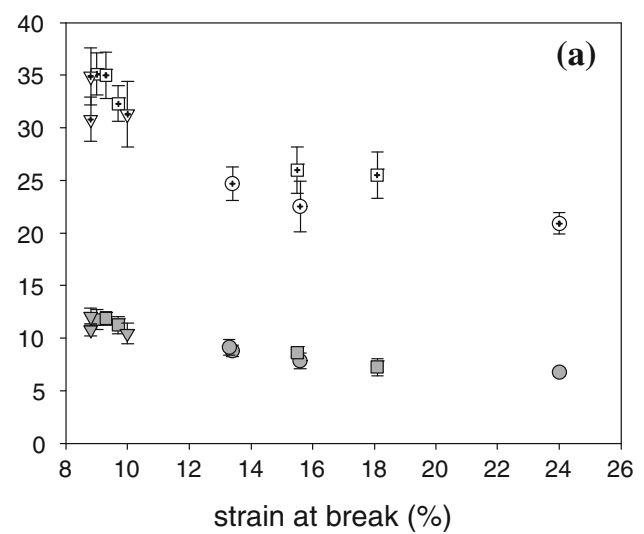

Fig. 7 Summary of results from mechanical analysis of airgap spun fibers from $15 \%$ pulp solutions. Graph (a) shows tenacity (cN/tex, white crosshair) and Young modulus, (N/tex, gray) as a function of strain at break. Graph (b) shows tenacity (cN/tex, white), strain at break (\%, black), and Young modulus (N/tex, dark gray) as a function

standard viscose in strength and to get strengths closer to lyocell and modal fibers stretching must be increased to the levels where elongation is limited to only $10 \%$. It appears that the amount of stretching necessary to reach this point is larger for the lower $x_{\mathrm{IL}}$ (with lower viscosity) solutions. Such an observation is quite expected when considering the lower viscosities, i.e., quicker relaxations of the solutions with more cosolvent. In those cases, the stresses and macromolecular structuring caused by elongational deformation do not persist until the coagulation point but are lost. It is also clear from the limited stretch in the case of $x_{\mathrm{IL}}=0.5$. Together, these facts mean that a very strong fiber cannot be achieved with a lot of cosolvent for the cellulose content tested. However, it appears that intermediate amounts of cosolvent can be used, as the drawability of the $x_{\mathrm{IL}}=0.7$ was reasonable and gave decent strengths over a wide range of stretch.

By recalculating the fiber properties for the neat EMIMAc samples (spun from $150 \mu \mathrm{m}$ spinneret), to what they would have been if they were spun from $80 \mu \mathrm{m}$ spinneret, all samples fall into the same trends, as seen in Fig. 7. Although generally giving lower strength than air-gap spinning, traditional wet spinning of EMIMAc-methylimidazole-cellulose solutions is very stable and allows for surprisingly large stretch, four times, both between spinneret and the first godet and between the first and second godet in stretch bath. This is an important difference to ordinary solutions of IL and cellulose which will die swell several times upon extrusion. If in the future, attempts are made to convert today's viscose plants to the use of closed loop-recycled solvent systems, low-viscosity wet spinning is what they are designed for. In that case, the use of cosolvents like methylimidazole might be important to the application of IL solvent systems in such plants. So, although the tenacities are not quite reaching commercial

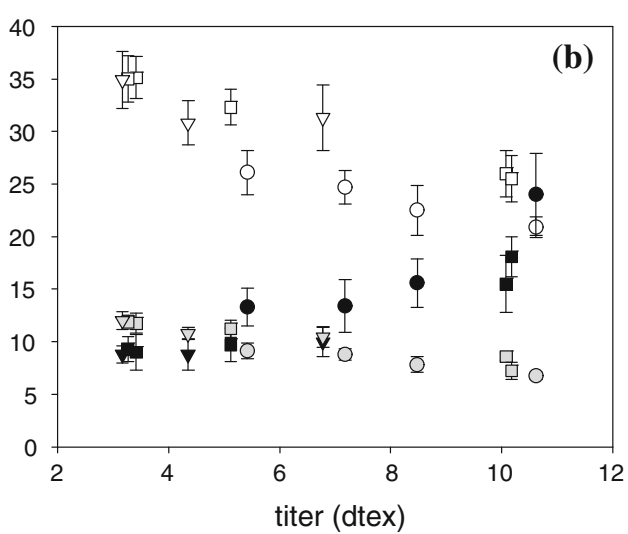

of fiber titer. All circles are $x_{\mathrm{IL}}=0.5$ samples, all squares are $x_{\mathrm{IL}}=0.7$ samples, and all triangles are $x_{\mathrm{IL}}=1.0$ samples (with the $x_{\mathrm{IL}}=1$ samples spun from $150 \mu \mathrm{m}$ capillaries normalized so that the titer will relate as the inverse to the stretch for the whole set of fibers spun, 10 dtex corresponding to no stretch)

levels yet, their further development might very well be worthwhile.

Crystallinity is claimed to be an important factor explaining fiber properties such as strength [38]. Hence, selected fibers were analyzed using solid state NMR, where a complete transformation from the crystal structure of native cellulose, cellulose I, could be noticed. As seen in Fig. 8, the peak at around $66 \mathrm{ppm}$, which is typical for a cellulose I crystal structure, is entirely missing in all regenerated samples. The crystallinity of the regenerated fibers is high and increases with increasing amount of cosolvent. This can be seen not only at around $107 \mathrm{ppm}$, where the crystal structure cellulose II has a characteristic peak, but also in the area between 84 and 90 ppm, which originates from $\mathrm{C} 4$ in the glucose ring. Peaks at 88.1 and

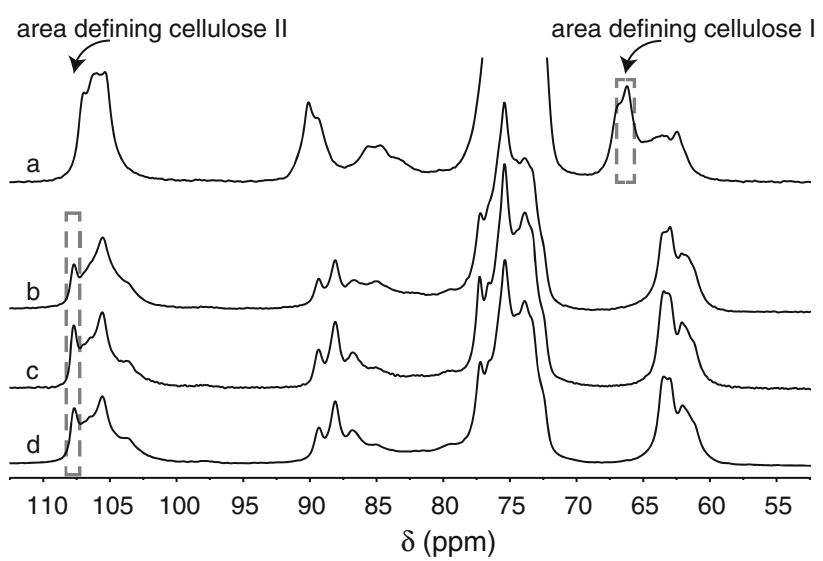

Fig. 8 Solid state NMR spectra for $(a)$ starting material dissolving pulp; and regenerated air gap spun fibers from different solvents (b) $x_{\mathrm{IL}}=1.0$ (pure EMIMAc), $(c) x_{\mathrm{IL}}=0.7$, and $(d) x_{\mathrm{IL}}=0.5$, all with the same applied stretch (two times). Increasing amount of cosolvent gives increasing crystallinity 
$89.3 \mathrm{ppm}$ are associated with cellulose II crystal interior atoms, while peaks at 86.6 and 85.6 are tentatively assigned to crystal surfaces [39, 40]. However, a broad gaussian peak at 84.2 originating from disordered material overlapping the crystalline signals complicates the interpretation of these data [41]. As the crystallinity of the fiber with only EMIMAc as solvent (Fig. 8b) is lower than for the fibers with co- solvent (Fig. 8c-d), it seems that in this case the crystallinity does not reflect the differences in tenacity.

\section{Recycling of solvent}

Recycling of the solvent is crucial in large scale processing in order to maintain sustainability and profitability, especially for costly solvents such as most ILs. In a process where cellulose is dissolved and subsequently precipitated and removed, as in a fiber-spinning process, the solvent will mix with the coagulation agent, for example, water. The boiling point of MIM is $198{ }^{\circ} \mathrm{C}$, and ILs have negligible vapor pressures at atmospheric pressure, which makes separation of water from the binary solvent mixture possible. Due to the thermal instability of EMIMAc [42], it is not possible to distill MIM from EMIMAc at the temperatures required at atmospheric pressure, as would be the case for a molecular solvent with lower boiling point. However, this problem can be circumvented either by reducing the pressure to a minimum, which would not be preferable on the large scale, or by simply purifying and reusing the IL-cosolvent mixture directly as previously proposed for very similar solvent systems [21, 25].

\section{Conclusions}

MIM as a cosolvent for cellulose solutions in ILs was evaluated, and it was shown that compared to other cosolvents in the study, it provides better solubility of cellulose and lower solution viscosity even at a very small amount of $\mathrm{IL}$ in the binary solvent. Moreover, the dissolution rate of cellulose increases dramatically using MIM. Fiber-spinning trials show that air-gap spinning can give good textile quality fibers if intermediate amounts of cosolvent are used. Wet spinning yields poorer results with regard to mechanical properties, but the ease with which these solutions are wetspun without any die swell and even allowing important amounts of jet-stretch is one of the most remarkable features of cosolvent addition to EMIMAc. Fibers from solvents including cosolvent show significantly higher crystallinity than fibers from pure IL solutions.
Further studies are currently being performed on the exact interactions among the cosolvent, IL, and cellulose and their implications on the potential applications of the solvent system and the regenerated material. A deeper understanding of the mechanisms that govern cellulose solubility is required to optimize solvent systems for different applications. The recycling procedure of solvent and cosolvent must be further improved, and it must be assured that their quality is maintained over a large number of cycles. Finally, the mechanisms on how to match the chosen cosolvent with a suitable IL in order to provide the desired advantages deserve further attention.

Acknowledgements This work was carried out within the framework of Avancell-Centre for Fibre Engineering, a research collaboration between Södra Innovation and Chalmers University of Technology. The NMR measurements were carried out at the Swedish NMR Centre, Göteborg, Sweden. Södra Skogsägarna Foundation for Research, Development and Education is gratefully being acknowledged for financial support.

Open Access This article is distributed under the terms of the Creative Commons Attribution License which permits any use, distribution, and reproduction in any medium, provided the original author(s) and the source are credited.

\section{Appendix}

See Figs. 9 and 10.

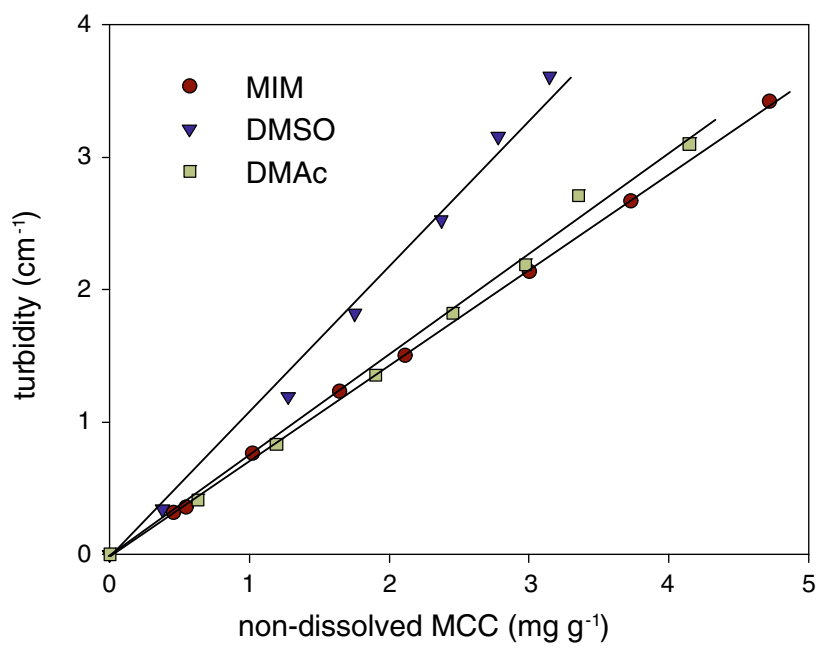

Fig. 9 Calibration curve of turbidity as a function of particular MCC in MIM, DMSO, and DMAc which shows excellent linearity $\left(\tau_{\mathrm{MIM}}=0.719 \cdot \mathrm{C}, \quad R^{2}=0.99 ; \quad \tau_{\mathrm{DMSO}}=1.101 \cdot \mathrm{C}, \quad R^{2}=0.99 ;\right.$ $\left.\tau_{\text {DMAc }}=0.753 \cdot \mathrm{C}, R^{2}=0.99\right)$ up to a turbidity value of at least $3 \mathrm{~cm}^{-1}$ 
Fig. 10 Complex viscosity of all spin dopes over a range of frequencies

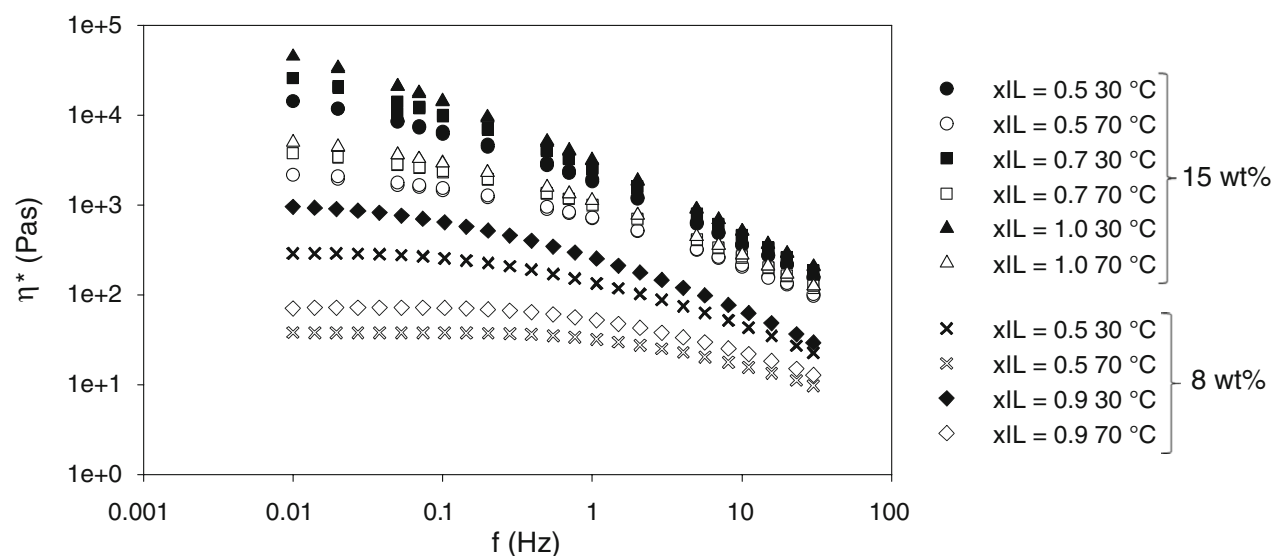

\section{References}

1. Lindman B, Karlström G, Stigsson L (2010) On the mechanism of dissolution of cellulose. J Mol Liq 156:76-81

2. Biermann O, Hädicke E, Koltzenburg S, Müller-Plathe F (2001) Hydrophilicity and lipophilicity of cellulose crystal surfaces. Angew Chem Int Ed 40:3822-3825

3. Meindersma GW, Maase M, Haan ABD (2007) Ionic liquids. In: Ullman Encyclopedia, (online encyclopedia) p. 1-33

4. Forsyth SA, Pringle JM, MacFarlane DR (2004) Ionic liquids: an overview. Aust J Chem 57:113-119

5. Zavrel M, Bross D, Funke M, Büchs J, Spiess AC (2009) Highthroughput screening for ionic liquids dissolving (ligno-) cellulose. Bioresour Technol 100:2580-2587

6. Hauru LKJ, Hummel M, King AWT, Kilpeläinen I, Sixta H (2012) Role of solvent parameters in the regeneration of cellulose from ionic liquid solutions. Biomacromolecules 13:2896-2905

7. Budtova T, Egal M, Gavillon R, Gericke M, Heinze T, Liebert T, Roy C, Schlufter K, Navard P (2010) Comparison of solutionstate properties of cellulose dissolved in $\mathrm{NaOH} /$ water and in ionic liquid (EMIMAc). In: Liebert T, Heinze T, Edgar KJ (eds) Cellulose solvents: for analysis, shaping and chemical modification. American Chemical Society, Washington, pp 179-196

8. Swatloski RP, Spear SK, Holbrey JD, Rogers RD (2002) Dissolution of cellose with ionic liquids. J Am Chem Soc 124: 4974-4975

9. Viswanathan G, Murugesan S, Pushpraj V, Nalamasu O, Ajayan PM, Linhardt RJ (2006) Preparation of biopolymer fibers by electrospinning from room temperature ionic liquids. Biomacromolecules 7:415-418

10. Kosan B, Michels C, Meister F (2008) Dissolution and forming of cellulose with ionic liquids. Cellulose 15:59-66

11. Wu J, Zhang J, Zhang H, He J, Ren Q, Guo M (2004) Homogeneous acetylation of cellulose in a new ionic liquid. Biomacromolecules 5:266-268

12. Heinze T, Schwikal K, Barthel S (2005) Ionic liquids as reaction medium in cellulose functionalization. Macromol Biosci 5:520-525

13. Kilpeläinen I, Xie H, King A, Granstrom M, Heikkinen S, Argyropoulus DS (2007) Dissolution of wood in ionic liquids. Agric Food Chem 55:9142-9148

14. Fort DA, Remsing RC, Swatloski RP, Moyna P, Rogers GMRD (2007) Can ionic liquids dissolve wood? Processing and analysis of lignocellulosic materials with 1-N-butyl-3-methylimidazolium chloride. Green Chem 9:63-69

15. Sun N, Rahman M, Qin Y, Maxim ML, Rodriguez H, Rogers RD (2009) Complete dissolution and partial delignification of wood in the ionic liquid 1-ethyl-3-methylimidazolium acetate. Green Chem 11:646-655

16. Heinze T, Dorn S, Schöbitz M, Liebert T, Köhler S, Meister F (2008) Interactions of ionic liquids with polysaccharides-2: cellulose. Macromol Sympos 262:8-22

17. Ebner G, Schiehser S, Potthast A, Rosenau T (2008) Side reaction of cellulose with common 1-alkyl-3-methylimidazolium-based ionic liquids. Tetrahedron Lett 49:7322-7324

18. Liebert $T$ (2008) Innovative concepts for the shaping and modification of cellulose. Macromol Symp 262:28-38

19. Froschauer C, Hummel M, Iakovlev M, Roselli A, Schottenberger H, Sixta H (2013) Separation of hemicellulose and cellulose from wood pulp by means of ionic liquid/cosolvent systems. Biomacromolecules 14:1741-1750

20. Gericke M, Liebert T, El SOA, Heinze T (2011) Tailored media for homogeneous cellulose chemistry: ionic liquid/co-solvent mixtures. Macromol Mater Eng 296:483-493

21. Gericke M, Schaller J, Liebert T, Fardim P, Meister F, Heinze T (2012) Studies on the tosylation of cellulose in mixtures of ionic liquids and a co-solvent. Carbohydr Polym 89:526-536

22. Gericke M, Liebert T, Heinze T (2009) Interaction of ionic liquids with polysaccharides, 8-synthesis of cellulose sulfates suitable for polyelectrolyte complex formation. Macromol Biosci 9:343-353

23. Härdelin L, Thunberg J, Perzon E, Westman G, Walkenström P, Gatenholm P (2012) Electrospinning of cellulose nanofibers from ionic liquids: the effect of different cosolvents. J Appl Polym Sci 125:1901-1909

24. Vitz J, Yevlampieva NP, Rjumtsev E, Schubert US (2010) Cellulose molecular properties in 1-alkyl-3-methylimidazoliumbased ionic liquid mixtures with pyridine. Carbohydr Polym 82:1046-1053

25. Rinaldi R (2011) Instantaneous dissolution of cellulose in organic electrolyte solutions. Chem Commun 47:511-513

26. Liu Z, Wang H, Li Z, Lu X, Zhang X, Zhang S, Zhou K (2011) Characterization of the regenerated cellulose films in ionic liquids and rheological properties of the solutions. Mater Chem Phys 128:220-227

27. Köhler S, Liebert T, Heinze T (2008) Interactions of ionic liquids with polysaccharides. VI pure cellulose nanoparticles from trimethylsilyl cellulose synthesized in ionic liquids. J Polym Sci Part A 46:4070-4080

28. Mormann W, Wezstein M (2009) Trimethylsilylation of cellulose in ionic liquids. Macromol Biosci 9:369-375

29. Gericke M, Schlufter K, Liebert T, Heinze T, Budtova T (2009) Rheological properties of cellulose/ionic liquid solutions: from dilute to concentrated states. Biomacromolecules 10:1188-1194 
30. Sescousse R, Le KA, Ries ME, Budtova T (2010) Viscosity of cellulose-imidazolium-based ionic liquid solutions. J Phys Chem B 114:7222-7228

31. Idström A, Brelid H, Nydén M, Nordstierna L (2013) CP/MAS ${ }^{13} \mathrm{C}$ NMR study of pulp hornification using nanocrystalline cellulose as a model system. Carbohydr Polym 92:881-884

32. Navard P, Wendler F, Meister F, Bercea M, Budtova T (2012) Preparation and properties of cellulose solutions. In: Navard P (ed) The European Polysaccharide Network of Excellence (EPNOE): research initiatives and results. Springer, Vienna, pp 91-152 Google eBook

33. Lovell CS, Walker A, Damion RA, Radhi A, Tanner SF, Budtova $\mathrm{T}$, Ries ME (2010) Influence of cellulose on ion diffusivity in 1-ethyl-3-methyl-imidazolium acetate cellulose solutions. Biomacromolecules 11:2927-2935

34. Kwei TK (1979) Macromolecules in solution. In: Bovey FA, Winslow FH (eds) Macromolecules. An introduction to polymer science. Academic Press, Inc, New York, pp 273-316

35. Le K, Sescousse R, Budtova T (2012) Influence of water on cellulose-EMIMAc solution properties: a viscometric study. Cellulose 19:45-54

36. Chen M, Bomble YJ, Himmel ME, Brady JW (2012) Molecular dynamics simulations of the interaction of glucose with imidazole in aqueous solution. Carbohydr Res 349:73-77
37. Cho HM, Gross AS, Chu J-W (2011) Dissecting force interactions in cellulose deconstruction reveals the required solvent versatility for overcoming biomass recalcitrance. J Am Chem Soc 133:14033-14041

38. Röder T, Moosbauer J, Wöss K, Schlader S, Kraft G (2013) Manmade cellulose fibres-a comparison based on morphology and mechanical properties. Lenzinger Berichte 91:7-12

39. Zuckerstätter G, Terinte N, Sixta H, Schuster KC (2013) Novel insight into cellulose supramolecular structure through $13 \mathrm{C} \mathrm{CP}$ MAS NMR spectroscopy and paramagnetic relaxation enhancement. Carbohydr Polym 93:122-128

40. Newman R, Davidson T (2004) Molecular conformations at the cellulose-water interface. Cellulose 11:23-32

41. Ibbett RN, Domvoglou D, Fasching M (2007) Characterisation of the supramolecular structure of chemically and physically modified regenerated cellulosic fibres by means of high-resolution carbon-13 solid-state NMR. Polymer 48:1287-1296

42. King AWT, Parviainen A, Karhunen P, Matikainen J, Hauru LKJ, Sixta H, Kilpelainen I (2012) Relative and inherent reactivities of imidazolium-based ionic liquids: the implications for lignocellulose processing applications. RSC Adv 2:8020-8026 